\title{
Single and multiple electron removal and fragmentation in collisions of protons with water molecules
}

\author{
L. Gulyás \\ Institute of Nuclear Research of the Hungarian Academy of Sciences (ATOMKI), PO Box 51, H-4001 Debrecen, Hungary \\ S. Egri \\ Department of Experimental Physics, University of Debrecen, 18/a Bem tér, H-4026 Debrecen, Hungary \\ H. Ghavaminia \\ Department of Physics, Dezful Branch, Islamic Azad University, Dezful, Iran
}

A. Igarashi

Faculty of Engineering, University of Miyazaki, Miyazaki 889-2192, Japan

(Received 18 December 2015; revised manuscript received 27 January 2016; published 8 March 2016)

\begin{abstract}
Single and multiple electron removal processes (capture and ionization) in proton- $\mathrm{H}_{2} \mathrm{O}$ collisions have been investigated applying the continuum distorted wave with eikonal initial-state model within the framework of independent electron approach. Probabilities and cross sections for electron capture are derived from the same quantities evaluated for ionization using the continuity of transition quantities across the ionization threshold. Dissociation and fragmentation cross sections for the $\mathrm{H}_{2} \mathrm{O}^{q+}(q=1-3)$ ions have been evaluated by considering branching ratios that include the effect of multiple electron removal transitions. The results are compared with experimental and other theoretical data in the range of impact energies from $30 \mathrm{kev}$ to $5 \mathrm{MeV}$. Generally, the evaluated cross sections and fragmentation yields show good agreement with experiments at impact energies above $100-150 \mathrm{keV}$.
\end{abstract}

DOI: 10.1103/PhysRevA.93.032704

\section{INTRODUCTION}

The interaction of charged particles with simple molecules is one of the most studied topics nowadays in molecular physics. In particular collisions of water molecules with ions are of fundamental importance not only in physics and astronomy but also in biology and medicine [1-3]. In recent years there has been a substantial development in the field of hadron therapy using proton or fast heavy ions [4]. About two-thirds of the human body is water and the production of low-energy electrons from $\mathrm{H}_{2} \mathrm{O}$ in an irradiated cell is of particular relevance because it is known that the thermalized electrons destroy very effectively large biomolecules in the tissue. Accurate knowledge of the intensity and distribution of the emitted electrons is especially important in the region of the Bragg peak [3]. Furthermore, an ionized water molecule can dissociate and the produced highly reactive species can further react within irradiated cells and provoke complex cellular damages [3,5,6]. In addition, for the various numerical models that have been implemented for simulating energy transport of energetic ions passing through biological tissues, water is considered as a good surrogate for biological medium, therefore, accurate knowledge of elementary processes in collisions of ions with $\mathrm{H}_{2} \mathrm{O}$ is of high relevance [7].

The widespread interest in reaction of water has led to a number of measurements for the total and differential cross sections for ionization and electron-capture mechanisms and to explore the fragmentation pathways of the ionized $\mathrm{H}_{2} \mathrm{O}$ molecules [8-16]. On the theoretical side, semiempirical methods have been used to evaluate the total and differential cross sections for single ionization of the $\mathrm{H}_{2} \mathrm{O}$ molecule [16,17]. Also, treatments based on the first Born $[9,18-20]$ and the continuum distorted-wave-eikonal initial state (CDW-EIS) [21-23] approximations have been applied to describe primary processes that happen in the collision of water with heavy ions. In all these treatments, within the framework of the independent electron picture, mostly single electron transitions are considered. A more accurate description of the various fragmentation channels can be given when multielectron transitions are also taken into account [24]. Multiple-ionization and electron-capture processes in ion- $\mathrm{H}_{2} \mathrm{O}$ collisions are considered in $[25,26]$ using the classical trajectory Monte Carlo (CTMC) treatment and in $[27,28]$ using the basis generator method (BGM).

In this work, we employ the CDW-EIS method to calculate transition probabilities and cross sections for singleand multiple-ionization processes in $\mathrm{H}^{+}-\mathrm{H}_{2} \mathrm{O}$ collisions in the impact energy range $0.03 \leqslant E_{P} \leqslant 5 \mathrm{MeV}$. In order to simplify the treatment, the multielectronic problem is reduced to the monoelectronic one using the independent electron approximation. The initial configuration of the molecule is described by multicenter wave functions provided by the GAUSSIAN program package [29]. The continuum state of the ejected electron has been given on an averaged spherical potential created by the spatially distributed passive electrons and nuclei. It should be noted that the description of the continuum orbital represents one of the main differences with the other applications of the CDW-EIS method [21,23] where the evaluation of the positive energy orbitals rely on Coulomb potential with some effective charge. Our model has already been applied in our previous study for the ionization of $\mathrm{CH}_{4}$ by proton impact [30]. A better description of molecular wave functions might substantially improve the agreement between theory and experiment even at the level total cross section. Of course the improvement might vary from target to target 


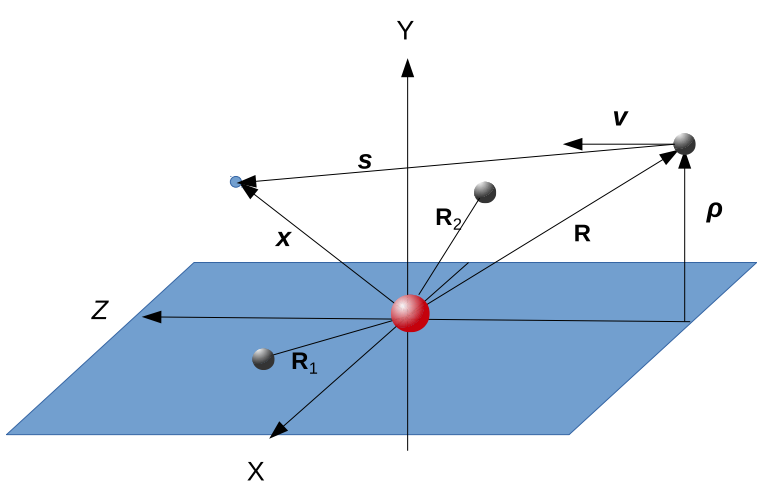

FIG. 1. Schematic of the $p-\mathrm{H}_{2} \mathrm{O}$ collision system.

which helps on the adjudication of the model and brings valuable knowledge that advances the field. Moreover, in the present application, not only the ionization of the molecule but electron capture by the projectile is also investigated. Electron-capture probabilities and cross sections are derived by using the continuity of ionization probabilities and cross sections across the ionization threshold. Fragmentation of the $\mathrm{H}_{2} \mathrm{O}$ molecule due to single or multiple electron removal is also considered. The paper is organized as follows: the CDW-EIS model for describing the proton- $\mathrm{H}_{2} \mathrm{O}$ collision is described in Sec. II. In Sec. III A total cross sections and probabilities for the ionization and electron-capture mechanism are presented. Multielectronic transitions and fragmentation of $\mathrm{H}_{2} \mathrm{O}$ are discussed in Sec. III B. The conclusion is drawn in Sec. IV.

Atomic units are used throughout unless indicated otherwise.

\section{THEORY}

Let us consider the case of bare nucleus of nuclear charge $Z_{P}$ impacting on the $\mathrm{H}_{2} \mathrm{O}$ molecule and as a result of the collision one or more electrons are removed from the target. As described in our previous work for the proton- $\mathrm{CH}_{4}$ collision system [30] we invoke the following three approximations in the theoretical description of the processes: (i) the impact parameter picture, where the incident particle is assumed to move on a straight line trajectory $\mathbf{R}=\rho+\mathbf{v} t$ with $\rho \perp \mathbf{v}$, where the constant velocity $\mathbf{v}$ is parallel to the $z$ axis of the laboratory system fixed at the center of the molecule (nucleus of the $\mathrm{O}$ atom for the present case) and $\rho \equiv\left(\rho, \varphi_{\rho}\right)$ denotes the impact parameter (see Fig. 1). (ii) fixed nuclei approximation, where the relative positions of nuclei in the molecule are treated as fixed during the collision; (iii) active-electron approximation, where only one electron is considered as active during the collision while all the remaining electrons have been described as frozen spectators. On the basis of these approximations, the single electron Hamiltonian is given by

$$
\begin{aligned}
h(\mathbf{x}, t) & =h_{0}(\mathbf{x})-\frac{Z_{P}}{|\mathbf{x}-\mathbf{R}(t)|}+V_{s}(\mathbf{R}(t)), \\
h_{0}(\mathbf{x}) & =-\frac{1}{2} \Delta_{\mathrm{x}}+v_{\text {molecule }}(\mathbf{x}),
\end{aligned}
$$

where $\mathbf{x}$ denotes the position vector of the active electron with respect to the target center (oxygen nucleus) (see Fig. 1), $h_{0}$ denotes the electronic Hamiltonian in the target molecule, and $v_{\text {molecule }}(\mathbf{x})$ describes the effective interaction of the electron with target nucleus and other electrons $\left(v_{\text {molecule }} \sim-1 / x\right.$ for $x \rightarrow \infty)$. The potential

$$
V_{s}(\mathbf{R}(t))=\left\langle\Phi_{p}\left|\sum_{j} \frac{Z_{P} Z_{j}}{\left|\mathbf{R}-\mathbf{R}_{j}\right|}-\sum_{k} \frac{Z_{P}}{s_{k}}\right| \Phi_{p}\right\rangle
$$

takes into account the interaction of the projectile with the target nuclei and other electrons, where $\mathbf{R}_{j}$ and $s_{k}$ stands for the position vector of the $j$ th nuclei and $k$ th passive electron relative to the projectile. $\Phi_{p}$ denotes collectively the wave function of the passive electrons that screen the nucleusnucleus interactions. Note that $V_{s} \sim Z_{P} / R$ for $R \rightarrow \infty$.

Before discussing the treatment for the active-electron dynamics, let us consider the description of the molecule. The symmetry of $\mathrm{H}_{2} \mathrm{O}$ is represented by the $C_{2 v}$ point group and the ground state of the molecule is described by the $\left(1 a_{1}\right)^{2}\left(2 a_{1}\right)^{2}\left(1 b_{2}\right)^{2}\left(3 a_{1}\right)^{2}\left(1 b_{1}\right)^{2}$ configuration $[31,32]$. In this study, only the four valence orbitals $\left(2 a_{1}, 1 b_{2}, 3 a_{1}\right.$, and $\left.1 b_{1}\right)$ are considered. Each valence orbital is constructed as a linear combination of atomic orbitals for the $\mathrm{O}$ and the two $\mathrm{H}$ atoms,

$$
\tilde{\Phi}_{i}\left(\mathbf{x}^{\prime}\right)=C_{O}^{i} \phi_{O}\left(\mathbf{x}^{\prime}\right)+\sum_{j=1}^{2} C_{H_{j}}^{i} \phi_{H_{j}}\left(\mathbf{x}^{\prime}-\mathbf{R}_{j}^{\prime}\right),
$$

where $i$ denotes any of the four valence orbitals, $\mathbf{R}_{j}^{\prime}$ is the position vector of the $j$ th $\mathrm{H}$ atom relative to the molecule center, and the primed quantities refer to the molecular frame. The $\phi_{\Xi}$ atomic orbitals have been constructed as contractions of Gaussian-type functions, using the STO-3G basis set [33]. Based on the Hartree-Fock approach the GAUSSIAN computational chemistry software package [29] has been used to obtain the $C_{\Xi}^{i}$ expansion coefficients. With this method we have obtained the following orbital energies: $-1.26,-0.617,-0.444$, and -0.3888 , respectively, for the $2 a_{1}, 1 b_{2}, 3 a_{1}$, and $1 b_{1}$ orbitals. Next, we expand (3) in terms of spherical harmonics,

$$
\tilde{\Phi}_{i}\left(\mathbf{x}^{\prime}\right)=\sum_{l_{i} \nu} u_{l_{i} \nu}(x) Y_{l_{i}}^{v}\left(\hat{\mathbf{x}}^{\prime}\right)
$$

where the coefficients are obtained by

$$
u_{l_{i} v}(x)=\int d \hat{\mathbf{x}}^{\prime}\left[Y_{l_{i}}^{v}\left(\hat{\mathbf{x}}^{\prime}\right)\right]^{*} \tilde{\Phi}_{i}\left(\mathbf{x}^{\prime}\right)
$$

$\tilde{\Phi}_{i}\left(\mathbf{x}^{\prime}\right)$ is written in the laboratory frame as

$$
\tilde{\Phi}_{i}\left(\mathbf{x}^{\prime}\right)=\Phi_{i, \alpha \beta \gamma}(\mathbf{x})=\sum_{l_{i} \nu} u_{l_{i}, \nu}(x) \sum_{m_{i}} Y_{l_{i}}^{m_{i}}(\hat{\mathbf{x}}) D_{l_{i}, \nu}^{m_{i}}(\alpha, \beta, \gamma),
$$

where $D_{l_{i}, \nu}^{m_{i}}(\alpha, \beta, \gamma)$ denotes the matrix element of the rotation operator for the rotation from the molecular to the laboratory frame through the Euler angles $(\alpha, \beta, \gamma)$.

The continuum states with energy $\left(\varepsilon_{k}=k^{2} / 2\right)$ and momentum $\mathbf{k}\left(k, \theta_{k}, \varphi_{k}\right)$ of the molecule, $\Phi_{\mathbf{k}}(\mathbf{x})$, is described on a spherically averaged potential created by the nuclei and the 
passive electrons [34],

$$
V_{\text {molecule }}^{+}(x)=V_{\text {nuclei }}(x)+V_{\text {electrons }}(x),
$$

where

$$
V_{\text {electrons }}(x)=-\sum_{i} n_{i} \frac{1}{4 \pi} \int d \mathbf{x}_{1} \frac{\left|\Phi_{i}\left(\mathbf{x}_{1}\right)\right|^{2}}{x_{>}}, \quad x_{>}: \max \left(x_{1}, x\right)
$$

and

$$
V_{\text {nuclei }}(x)= \begin{cases}-\frac{Z_{o}+2 Z_{H}}{x} & \text { if } x>R_{o}, \\ -\frac{Z_{O}}{x}-\frac{2 Z_{H}}{R_{o}} & \text { if } x<R_{o},\end{cases}
$$

where $Z_{O}$ and $Z_{H}$ are the nuclear charges of the $\mathrm{O}$ and $\mathrm{H}$ atoms. $R_{o}$ is the radius of a sphere around the oxygen nucleus representing an averaged uniform spherical charge distribution for the nucleus of $\mathrm{H}$ atoms. As for the initial orbital [see Eq. (4)], $\Phi_{\mathbf{k}}(\mathbf{x})$ is expanded over spherical harmonics,

$$
\Phi_{\mathbf{k}}(\mathbf{x})=\frac{1}{x \sqrt{k}} \sum_{l, m} i^{l} e^{-i \delta_{l}} u_{k l}(x)\left[Y_{l}^{m}(\hat{\mathbf{x}})\right]^{*} Y_{l}^{m}(\hat{\mathbf{k}}),
$$

where $u_{k l}(x)$ is obtained on the numerical solution for the radial part of the molecular Hamiltonian: $h_{\text {molecule }}=-\frac{1}{2} \Delta_{\mathrm{x}}+$ $V_{\text {molecule }}^{+}(x)$; see [35].

The above description of molecular wave functions allows us to describe the collision dynamic within the framework of the CDW-EIS model [30]. The CDW-EIS model is a first-order method which proved to be very successful for describing atomic collisions at medium and high impact energies $[36,37]$ and its extension to molecular collisions have been provided in $[21-23,30]$. The effect of the projectile on the electronic state is taken into account by using the eikonal distorted-wave function,

$$
\begin{aligned}
& \chi_{i, \alpha \beta \gamma}^{+}(\mathbf{x}, t)=\exp \left(-i \int_{-\infty}^{t} V_{s}\left(t^{\prime}\right) d t^{\prime}\right) \xi_{i, \alpha \beta \gamma}^{+}(\mathbf{x}, t), \\
& \xi_{i, \alpha \beta \gamma}^{+}(\mathbf{x}, t)=e^{-i \varepsilon_{i} t} \Phi_{i, \alpha \beta \gamma}(\mathbf{x}) E_{\mathbf{v}}^{*}\left(\mathbf{s}, \eta_{i}\right),
\end{aligned}
$$

for the initial channel, and by the Coulomb distorted-wave function,

$$
\begin{aligned}
& \chi_{\mathbf{k}}^{-}(\mathbf{x}, t)=\exp \left(i \int_{t}^{+\infty} V_{s}\left(t^{\prime}\right) d t^{\prime}\right) \xi_{\mathbf{k}}^{-}(\mathbf{x}, t), \\
& \xi_{\mathbf{k}}^{-}(\mathbf{x}, t)=e^{-i \varepsilon_{k} t} \Phi_{\mathbf{k}}^{-}(\mathbf{x}) D_{\mathbf{p}}\left(\mathbf{s}, \eta_{P}\right)
\end{aligned}
$$

for the ionization channel. The distortion factors $D_{\mathbf{p}}\left(\mathbf{s}, \eta_{P}\right)$ and $E_{\mathbf{v}}^{*}\left(\mathbf{s}, \eta_{i}\right)$ are given by

$$
D_{\mathbf{p}}\left(\mathbf{s}, \eta_{p}\right)=e^{\pi \eta_{p} / 2} \Gamma\left(1+i \eta_{p}\right)_{1} F_{1}\left(-i \eta_{p}, 1,-i(p s+\mathbf{p} \cdot \mathbf{s})\right)
$$

and

$$
E_{\mathbf{v}}\left(\mathbf{s}, \eta_{i}\right)=(v s+\mathbf{v} \cdot \mathbf{s})^{i \eta_{i}},
$$

respectively, where $\eta_{i}=Z_{P} / v, \eta_{P}=Z_{P} / p, \mathbf{p}=\mathbf{k}-\mathbf{v}, \mathbf{s}=$ $\mathbf{x}-\mathbf{R}$, and ${ }_{1} F_{1}$ is the confluent hypergeometric function. It is interesting to note that for a large argument the eikonal phase $E_{\mathbf{u}}(\mathbf{r}, \eta)$ is the asymptotic form of the Coulomb distortion function $D_{\mathbf{u}}(\mathbf{r}, \eta)$. The CDW-EIS model is considered as a simplified version of the full or symmetric CDW method where
Coulomb distortions are employed in both initial and final channels [36]. The justification of using eikonal distortion is related to incorrect normalization of the initial state with the Coulomb distortion factor, which results in overestimation of cross sections at low impact energies [38,39]. The distorted wave functions (11) and (12) satisfy the correct boundary conditions,

$$
\chi_{i, \alpha \beta \gamma}^{+}(\mathbf{x}, t \rightarrow-\infty) \sim e^{-i \varepsilon_{i} t} \Phi_{i, \alpha \beta \gamma}(\mathbf{x})
$$

and

$$
\begin{aligned}
\chi_{\mathbf{k}}^{-} \sim & \sqrt{\frac{k}{(2 \pi)^{3}}} e^{-i \varepsilon_{k} t} e^{i \mathbf{k} \cdot \mathbf{x}} \\
& \times(k x+\mathbf{k} \cdot \mathbf{x})^{i / k}(p s+\mathbf{p} \cdot \mathbf{s})^{i \eta_{p}}(v R+\mathbf{v} \cdot \mathbf{R})^{-i \eta_{i}},
\end{aligned}
$$

for $x \rightarrow \infty, s \rightarrow \infty$, and $t \rightarrow+\infty$. Hence the wave functions (11) and (12) fulfill requirements settled for atomic or pure three-body Coulomb collision systems $[36,40]$. Beyond the correct asymptotic behavior, the CDW wave function describes the active electron's moving in the combined electric field created by the projectile and target core. This is also an important character of the theory and known as two center effects [37].

The transition amplitude in prior form can be written as

$$
\begin{aligned}
\mathcal{A}_{i \mathbf{k}}^{-}\left(\boldsymbol{\rho}, \omega_{E}\right) & =-i \int_{-\infty}^{\infty} d t\left\langle\chi_{\mathbf{k}}^{-}(\mathbf{x}, t) \mid\left(h(\mathbf{x}, t)-i \frac{\partial}{\partial t}\right) \chi_{i \omega_{E}}^{+}(\mathbf{x}, t)\right\rangle \\
& =\exp \left(-i \int_{-\infty}^{+\infty} V_{s}\left(t^{\prime}\right) d t^{\prime}\right) a_{i \mathbf{k}}^{-}\left(\boldsymbol{\rho}, \omega_{E}\right), \\
a_{i \mathbf{k}}^{-}\left(\boldsymbol{\rho}, \omega_{E}\right) & =-i \int_{-\infty}^{\infty} d t\left\langle\xi_{\mathbf{k}}^{-}(\mathbf{x}, t) \mid\left(\tilde{h}(\mathbf{x}, t)-i \frac{\partial}{\partial t}\right) \xi_{i \omega_{E}}^{+}(\mathbf{x}, t)\right\rangle
\end{aligned}
$$

with $\omega_{E}=\{\alpha, \beta, \gamma\}, \tilde{h}=h-V_{s}$, and

$$
\begin{aligned}
\left(\tilde{h}-i \frac{\partial}{\partial t}\right) \xi_{i, \alpha \beta \gamma}^{+}(\mathbf{x}, t)= & -e^{-i \varepsilon_{i} t}\left[\Phi_{i, \alpha \beta \gamma}(\mathbf{x}) \frac{1}{2} \nabla_{\mathbf{x}}^{2} E_{\mathbf{v}}^{*}\left(\mathbf{s}, \eta_{i}\right)\right. \\
& \left.+\nabla_{\mathbf{x}} \Phi_{i, \alpha \beta \gamma}(\mathbf{x}) \cdot \nabla_{\mathbf{s}} E_{\mathbf{v}}^{*}\left(\mathbf{s}, \eta_{i}\right)\right] .
\end{aligned}
$$

The probability for the electron emission with energy $\varepsilon_{k}$ in the direction of $d \Omega_{k}\left(\theta_{k}, \phi_{k}\right)$ from a given $i$ th initial orbital is given by

$$
\frac{d^{2} p_{i}(\boldsymbol{\rho})}{d \varepsilon_{k} d \Omega_{k}}=\left|A_{i \mathbf{k}}^{-}\left(\boldsymbol{\rho}, \omega_{E}\right)\right|^{2}=\left|a_{i \mathbf{k}}^{-}\left(\boldsymbol{\rho}, \omega_{E}\right)\right|^{2},
$$

and the phase factor in Eq. (17), $\exp \left(-i \int_{-\infty}^{+\infty} V_{s}\left(t^{\prime}\right) d t^{\prime}\right)$, does not affect the probability within the CDW-EIS model. In CDW models it is more convenient to evaluate the Fourier transform of $a_{i \mathbf{k}}^{-}\left(\boldsymbol{\rho}, \omega_{E}\right)$,

$$
\begin{aligned}
f_{i \mathbf{k}}\left(q, \varphi_{q}-\varphi_{k}, \omega_{E}\right)= & \frac{1}{2 \pi} \int d \boldsymbol{\rho} e^{i \mathbf{q} \cdot \boldsymbol{\rho}} a_{i \mathbf{k}}^{-}\left(\boldsymbol{\rho}, \omega_{E}\right) \\
= & \sum_{l_{i} \nu m_{i}} D_{l_{i}, v}^{m_{i}}(\alpha, \beta, \gamma) \sum_{l m \mu}\left[Y_{l}^{m}(\hat{\mathbf{k}})\right]^{*} e^{i\left(m+m_{i}\right) \varphi_{q}} \\
& \times \mathcal{R}_{l_{i} \nu m_{i}}^{l m \mu}\left(q, k, \theta_{k}\right) e^{i \mu\left(\varphi_{q}-\varphi_{k}\right)}
\end{aligned}
$$


with $\mathbf{q}=\left(q \cos \varphi_{q}, q \sin \varphi_{q}, 0\right)$. Then, $a_{i \mathbf{k}}^{-}$can be obtained by the inverse Fourier transform $[41,42]$,

$$
\begin{aligned}
a_{i \mathbf{k}}^{-}\left(\boldsymbol{\rho}, \omega_{E}\right)= & \frac{1}{2 \pi} \int d \mathbf{q} e^{-i \mathbf{q} \cdot \boldsymbol{\rho}} f_{i \mathbf{k}}\left(q, \varphi_{q}-\varphi_{k}, \omega_{E}\right) \\
= & \sum_{l_{i} \nu m_{i}} D_{l_{i}, v}^{m_{i}}(\alpha, \beta, \gamma) \sum_{l m \mu}(-i)^{\mu+M} \mathcal{Y}_{l}^{m}(\hat{\mathbf{k}}) \\
& \times e^{-i \mu \varphi_{k}+i(\mu+M) \varphi_{\rho}} \int d q q J_{\mu}(q \rho) \mathcal{R}_{l_{i} \nu m_{i}}^{l m \mu}\left(q, k, \theta_{k}\right),
\end{aligned}
$$

where $M=m+m_{i}, \mathcal{Y}_{l}^{m}(\hat{\mathbf{k}})=Y_{l}^{m}(\hat{\mathbf{k}}) \exp \left(-i m \varphi_{k}\right)$, and $J_{\mu}$ stands for the Bessel function; see $[35,41,42]$ for more details.

The molecules have an arbitrary orientation in the experiments discussed in the next section, and we average the probability in Eq. (19) over the Euler angles and $\varphi_{\rho}$,

$$
\frac{d^{2} p_{i}(\rho)}{d \varepsilon_{k} d \Omega_{k}}=\frac{1}{8 \pi^{2}} \int d \omega_{E} \frac{1}{2 \pi} \int d \varphi_{\rho} \frac{d^{2} p_{i}(\boldsymbol{\rho})}{d \varepsilon_{k} d \Omega_{k}},
$$

where the integral over Euler angles $\left(\int d \omega_{E}=\right.$ $\left.\int_{0}^{2 \pi} d \alpha \int_{0}^{\pi} d \beta \sin \beta \int_{0}^{2 \pi} d \gamma\right)$ is analytic owing to the orthogonal property of $D_{l, \mu}^{m}$ [43].

Single-particle ionization probability is obtained by

$$
p_{i}(\rho)=\int d \varepsilon_{k} \int d \Omega_{k} \frac{d^{2} p_{i}(\rho)}{d \varepsilon_{k} d \Omega_{k}} .
$$

For a target with $N$ electrons, the integrated net ionization probability can be defined by

$$
P_{\text {net }}(\rho)=\sum_{i=1}^{N} p_{i}(\rho) .
$$

This quantity corresponds to the average number of emitted electrons and is the mean value of the distribution of the probabilities $P_{q}$ for the ionization of $q$ out of $N$ electrons,

$$
P_{\text {net }}(\rho)=\sum_{q=1}^{N} q P_{q}(\rho) .
$$

In the framework of the independent particle model (IPM), the probabilities $P_{q}$ for $q$-fold ionization are calculated by a binomial analysis of the single-particle probabilities $p_{i}$. On the level of a shell-specific model [44] $P_{q}$ is given by

$$
\begin{aligned}
P_{q}(\rho)= & \sum_{\substack{q_{1}, \ldots, q_{m}=0 ; q_{1}+\cdots+q_{m}=q \\
N_{1}, \ldots, N_{m}}} \prod_{i=1}^{m} \frac{N_{i} !}{q_{i} !\left(N_{i}-q_{i}\right) !}\left[p_{i}(\rho)\right]^{q_{i}} \\
& \times\left[1-p_{i}(\rho)\right]^{N_{i}-q_{i}} .
\end{aligned}
$$

Here, $m$ is the number of electron shells, and $N_{i}$ is the number of electrons in each shell. Total cross sections (TCSs) for $q$-fold ionization are obtained by integration of $\rho P_{q}(\rho)$ over the impact parameter $\rho$.

Among the various ionization processes [37] the mechanism of electron capture into the projectile's continuum (ECC) presents a special character. Due to the continuity of the probability above the ionization limit this process relates to the capture into highly excited $\mathrm{H}(n)$ levels just below the ionization threshold [45-47], which can be expressed as

$$
\lim _{\varepsilon_{e}^{\prime} \rightarrow 0} \frac{d p(\rho)}{d \varepsilon_{e}^{\prime}}=\lim _{n \rightarrow \infty} \frac{d p_{n}(\rho)}{d \varepsilon_{n}} \equiv \lim _{n \rightarrow \infty} n^{3} \frac{d p_{n}(\rho)}{d n},
$$

where $d p(\rho) / d \varepsilon_{e}^{\prime}$ is the single-ionization probability versus electron emission energy and now the prime indicates the quantities of electron emission seen in the projectile frame [48]. Furthermore, at high collision energies $p_{n}(\rho)$ scales as $p_{n}(\rho)=p_{1}(\rho) n^{-3}[40]$, which enables us to evaluate the probability (and so cross section) for capture of an electron even into the $n=1$ level.

The above single and multiple transition probabilities and cross sections correspond to the physical stage of the collision where molecular excitations and ionizations take place [49]. The physical stage is followed by the physicochemical stage which includes the fast rearrangement and dissociation of the molecule.

\section{RESULTS}

In this section results for single and multiple ionization, single and multiple electron capture, and dissociation of $\mathrm{H}_{2} \mathrm{O}$ induced by proton impact in the $0.01-5-\mathrm{MeV}$ impact energy range are presented. As for the treatment of $\mathrm{CH}_{4}$ [30], the expansion of $\tilde{\Phi}_{i}\left(\mathbf{x}^{\prime}\right)$ in Eq. (4) is performed only for limited values of $l_{i}\left(l_{i}=0,1 \rightarrow l_{i}^{\max }\right)$. The cross-section data have been found to converge when $l_{i}^{\max } \geqslant 3$ for all the reported collision energies. As in [30], the equilibrium $\mathrm{O}-\mathrm{H}$ distance $\left(R_{o}\right)$ has been reduced by $30 \%$ during evaluation of the continuum orbital in order to decrease the attractive force of the $\mathrm{H}$ nuclei around the $\mathrm{O}$ center. In the following discussions we refer to the model described above as CDW-EIS-MO.

\section{A. Total cross sections and probabilities}

In Fig. 2 net ionization and capture probabilities as a function of impact parameter for the collision of $\mathrm{H}_{2} \mathrm{O}$ with 100and 500-keV projectile energies are shown. The CDW-EISMO capture probabilities are evaluated along the formula (27), where capture into excited levels is also included [50]. For the case of ionization, we also apply a method, where the transition probability for a given molecular orbital is obtained as a weighted sum of atomic probabilities corresponding to the atomic constituents of the molecule [51,52]:

$$
p^{\mathrm{H}_{2} \mathrm{O}}=p_{2 a_{1}}^{\mathrm{H}_{2} \mathrm{O}}+p_{1 b_{2}}^{\mathrm{H}_{2} \mathrm{O}}+p_{3 a_{1}}^{\mathrm{H}_{2} \mathrm{O}}+p_{1 b_{1}}^{\mathrm{H}_{2} \mathrm{O}}
$$

with

$$
\begin{gathered}
p_{2 a_{1}}^{\mathrm{H}_{2} \mathrm{O}}=1.48 p_{2 s}^{\mathrm{O}}+0.52 p_{1 s}^{\mathrm{H}}, \\
p_{1 b_{2}}^{\mathrm{H}_{2} \mathrm{O}}=1.18 p_{2 s}^{\mathrm{O}}+0.82 p_{1 s}^{\mathrm{H}}, \\
p_{3 a_{1}}^{\mathrm{H}_{2} \mathrm{O}}=0.22 p_{2 s}^{\mathrm{O}}+1.44 p_{2 p}^{\mathrm{O}}+0.34 p_{1 s}^{\mathrm{H}}, \\
p_{1 b_{1}}^{\mathrm{H}_{2} \mathrm{O}}=2.00 p_{2 p}^{\mathrm{O}},
\end{gathered}
$$

where $p$ is the total ionization probability of (23), the lower index indicates initial orbital quantum numbers of a given atom or molecule (denoted be upper index), and the expansion coefficients were derived in self-consistent field molecular calculation $[31,53]$. This method, based on atomic components, is 

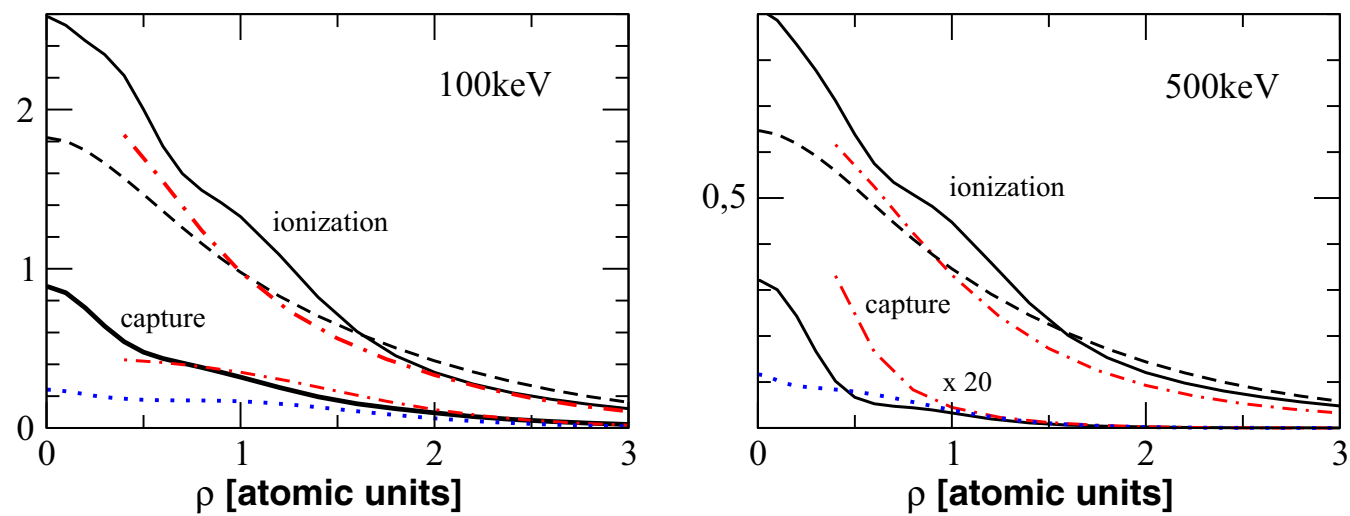

FIG. 2. Net ionization and capture probabilities as a function of impact parameter for the $p-\mathrm{H}_{2} \mathrm{O}$ collision system at $E_{p}=100-$ and 500-keV impact energies. Present calculations: CDW-EIS-MO (solid lines), CDW-EIS-AC (dashed lines), and E-AC (dotted lines). BGM from [54] (dot-dashed lines). For the right panel (500 keV) all capture probabilities are multiplied by a factor of 20.

referred to hereafter as CDW-EIS-AC. Although the structure of the molecule is neglected in this model and so the meaning of a direct comparison with the MO treatment is limited, we found it interesting to present it in the figure as CDW-EIS-AC provides very realistic values for cross sections (integrated probabilities over $\rho$ ); see Fig. 3. It can be observed that the ionization probabilities by the CDW-EIS-MO are larger than those by CDW-EIS-AC at low $\rho$ values and this tendency changes at $\rho \approx 2$, and at the asymptotic region the CDWEIS-AC model predicts the larger probabilities. Figure 2 also presents results of Basis Generator Method (BGM) by Lüdde et al. [54], which shows good agreement with the present data except for the case of capture at $500 \mathrm{keV}$ impact energy. Capture probabilities derived as for CDW-EIS-AC but eikonal approximation for the atomic transitions (E-AC) [50] are also shown, which reveal a satisfactory account of the process only at $500 \mathrm{keV}$ impact energy. The probability distributions evaluated in CDW-EIS-MO show a small hump at $\rho \approx 1$, which is mostly attributed to contributions of $3 a_{1}$ and $1 b_{1}$ orbitals. The hump has been found less pronounced in some test calculations where the $\mathrm{O}-\mathrm{H}$ distance is reduced, however it does not disappear even when the $\mathrm{H}$ atoms were placed at the

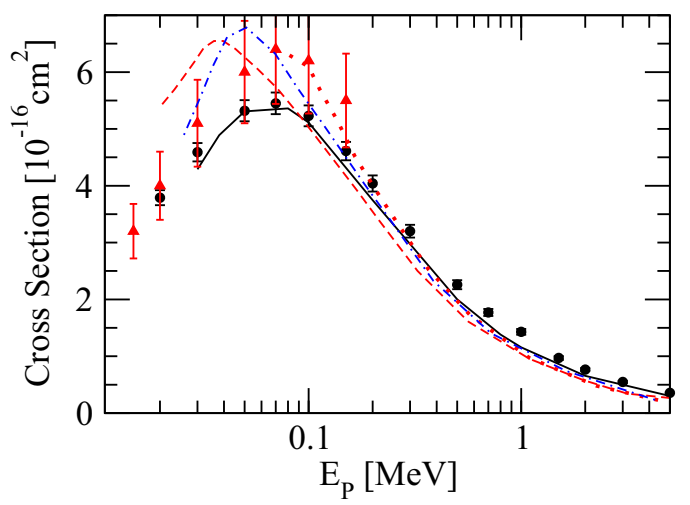

FIG. 3. Net ionization cross sections as a function of the projectile energy for the $p-\mathrm{H}_{2} \mathrm{O}$ collision system. Theoretical calculations, solid line: present CDW-EIS-MO; dashed line: BGM of Ref. [28]; dotdashed line: CTMC of Ref. [26]; dotted line: CDW-EIS of Ref. [22] Experiments: $(\bullet)$, from [11]; $(\triangle)$ from [12]. molecule center. Probabilities evaluated for the $\mathrm{O}$ and $\mathrm{H}$ atoms [see (29)-(32)] do not reveal any structure over the full range of $\rho$ and obviously the CDW-EIS-AC and E-AC methods do not predict any hump in the probability distribution. Interestingly, this structure is also missing in the results of BGM calculation, where the geometrical arrangement of the molecule has also been taken into account.

The net ionization cross sections are plotted as a function of impact energy in Fig. 3. The figure shows the present CDE-EIS-MO cross sections in comparison with results of the BGM [54], CDW-EIS [22], and classical trajectory Monte Carlo (CTMC) [26] calculations and with the available experimental data. The present CDW-EIS-AC results are not plotted in the figure as they are almost the same as those of CDW-EIS-MO. The present calculations show nice agreement with results of the other calculations at the highest impact energies and with the experiment of [11] in the full range of impact energy. Discrepancies between theories appear at medium impact energies, where the present cross sections are lower than predictions of the other methods. Also the experimental data by [12] are higher than those from [11] at medium impact energies, but with higher error bars that do not help the adjudication of theories.

Cross sections for net electron capture are presented in Fig. 4. Present capture cross sections, evaluated by the continuation of the ECC ionization cross sections across the threshold [see Eq. (27)], show very good agreement with the experimental data. Although the reliability of the method has been verified in other studies [48,56] the nice account of the experimental results at the low impact energies is unexpected. We have also applied the CDW method for capture [57] to get the capture cross sections from the $\mathrm{O}$ and $\mathrm{H}$ atoms and have evaluated capture cross sections for $\mathrm{H}_{2} \mathrm{O}$ on the basis of atomic components as for the case of ionization; see Eqs. (28)-(32). These cross sections agree nicely with the CDW-EIS-MO cross sections evaluated along the continuation of ECC quantities across the threshold [see Eq. (27)] and differences appear only at the low impact energies. As for the case of ionization, the agreement between these two sets of cross sections underlines the minor importance of the description of molecular orbitals for the process of capture too. The increasing discrepancy at lower impact energies can 


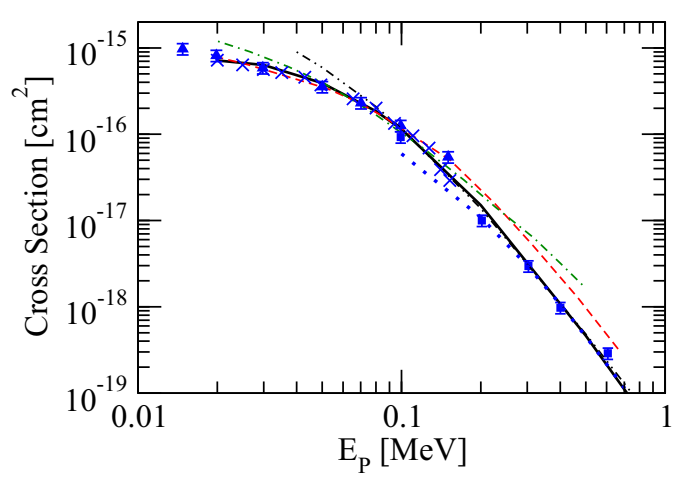

FIG. 4. Cross section for net electron capture from $\mathrm{H}_{2} \mathrm{O}$ molecule by proton impact. Theory: solid line, present CDW-EIS-MO; dot-dotdashed line present CDW result for capture using the atomic statistic; dotted line: present E-AC; dashed line BGM from [27]; dot-dashed line CTMC of Ref. [26]. Experiments: $(\triangle)$ from [11]; $(\square)$ from [55] (x) from [13].

be explained by the distinct validity of the different CDW methods. The CDW method suffers from normalization failure of the scattering wave functions which results in unrealistically high cross sections at low impact energies [36,50]. This failure is corrected in the CDW-EIS method which provides more realistic results than the CDW at low projectile velocities [39]. This explains the deviation between the two CDW treatments at the low impact energy region. We note that in [58] the CDWEIS model with the atomic constituents of the molecule [see Eqs. (28)-(32)] have been applied for evaluating capture cross sections. Their results, not shown in the figure, are slightly higher than the present one, especially at low impact energies, which we think is mostly related to the different description of the atomic transition quantities. The present E-AC results are also given in the figure, which shows good results at above 200 $\mathrm{keV}$ impact energies. The figure also presents the BGM results from [27] which show good agreement with the experimental data at low impact energies and CTMC calculations from [26] showing good results only at a limited region of projectile energies.

\section{B. Multiple electron removal and fragmentation}

In addition to the process of ionization, electron(s) can also be removed from the $\mathrm{H}_{2} \mathrm{O}$ molecule by the charge-transfer mechanism. Production of electron vacancy in the target (due to ionization or capture) is referred to as electron loss. Multiple electron transitions are evaluated in the framework of IPM, where $q$-fold electron transitions are calculated by the binomial statistic of single-particle transitions [30,59]. Cross sections for $q$-fold electron loss are plotted as a function of impact energy in Fig. 5. The figure shows the present CDW-EIS-MO cross sections in comparison with results of the BGM calculation from [27]. Good agreement can be observed for $q=1$ and 3, while a slight discrepancy appears for the case of $q=2$ at higher impact energies (the discrepancy will be discussed below). The figure also shows the present CDWEIS-MO cross sections for the case of multiple ionization. Considerable differences can be observed at the low impact energies, where the $q$-fold loss cross sections are much higher

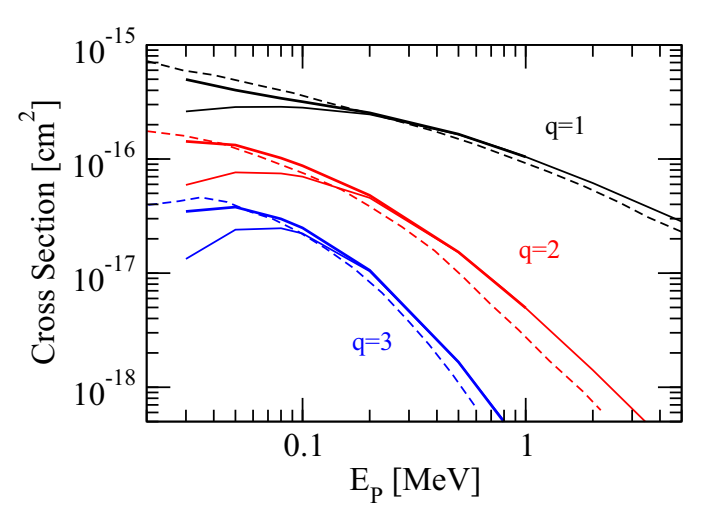

FIG. 5. Theoretical cross sections for $q$-fold electron loss and ionization of water by fast proton impact. Results for electron loss; heavy solid line: present CDW-EIS-MO, dashed line: BGM calculations from [27]. Thin solid lines are present CDW-EIS-MO results for ionization.

than those of pure $q$-fold ionization. Obviously the difference is due to the contribution of the capture process which becomes less important with the increase of projectile energy and so much better agreement can be realized at high impact energies.

Removing an electron from the water molecule leads to the breakdown of the $\mathrm{H}_{2} \mathrm{O}^{+}$ion and besides the $\mathrm{H}_{2}{ }^{+}$and $\mathrm{O}^{2+}$ fragments $\mathrm{OH}^{+}, \mathrm{H}^{+}$, and $\mathrm{O}^{+}$ions are produced in measurable amounts. Fragmentation cross sections for the singly charged ions can be estimated on the basis of empirical branching ratios of Tan et al. [15]. However, as it was found in [27] the fragmentation pattern of [15] is not very useful at low impact energies and the extension of the model by including contributions from the double- and triple-ionized $\mathrm{H}_{2} \mathrm{O}$ molecules was proposed for the fragmentation cross sections:

$$
\begin{gathered}
\sigma_{H_{2} O^{+}}=0.68 \sigma_{S}, \\
\sigma_{O H^{+}}=0.16 \sigma_{S}+0.6 \sigma_{D}, \\
\sigma_{H^{+}}=0.13 \sigma_{S}+1.2 \sigma_{D}+1.0 \sigma_{T}, \\
\sigma_{O^{+}}=0.03 \sigma_{S}+0.2 \sigma_{D}+0.5 \sigma_{T},
\end{gathered}
$$

where $\sigma_{S}, \sigma_{D}$, and $\sigma_{T}$ refer to single, double, and triple electron ionization or removal cross sections as presented in Fig. 5. Figure 6 presents fragmentation cross sections [see (33)-(36)] for the dissociation of collision induced single, double, and triple charged $\mathrm{H}_{2} \mathrm{O}$ ions in comparison with the available experimental data. Reasonable agreement with experimental data, both for ionization and loss induced fragmentations, can be observed at high impact energies, however discrepancies appear below 50-100-keV impact energies for all fragment ions. As expected, the fragmentation yields at high impact energies are dominated by dissociation of ions formed in the process of ionization as the capture mechanism presents negligible contributions. The figure presents also fragmentation cross sections evaluated from the electron-loss cross sections along the formulas (33)-(36) from [27] which reveal nearly the same accounts of the experimental data as the present ones. 

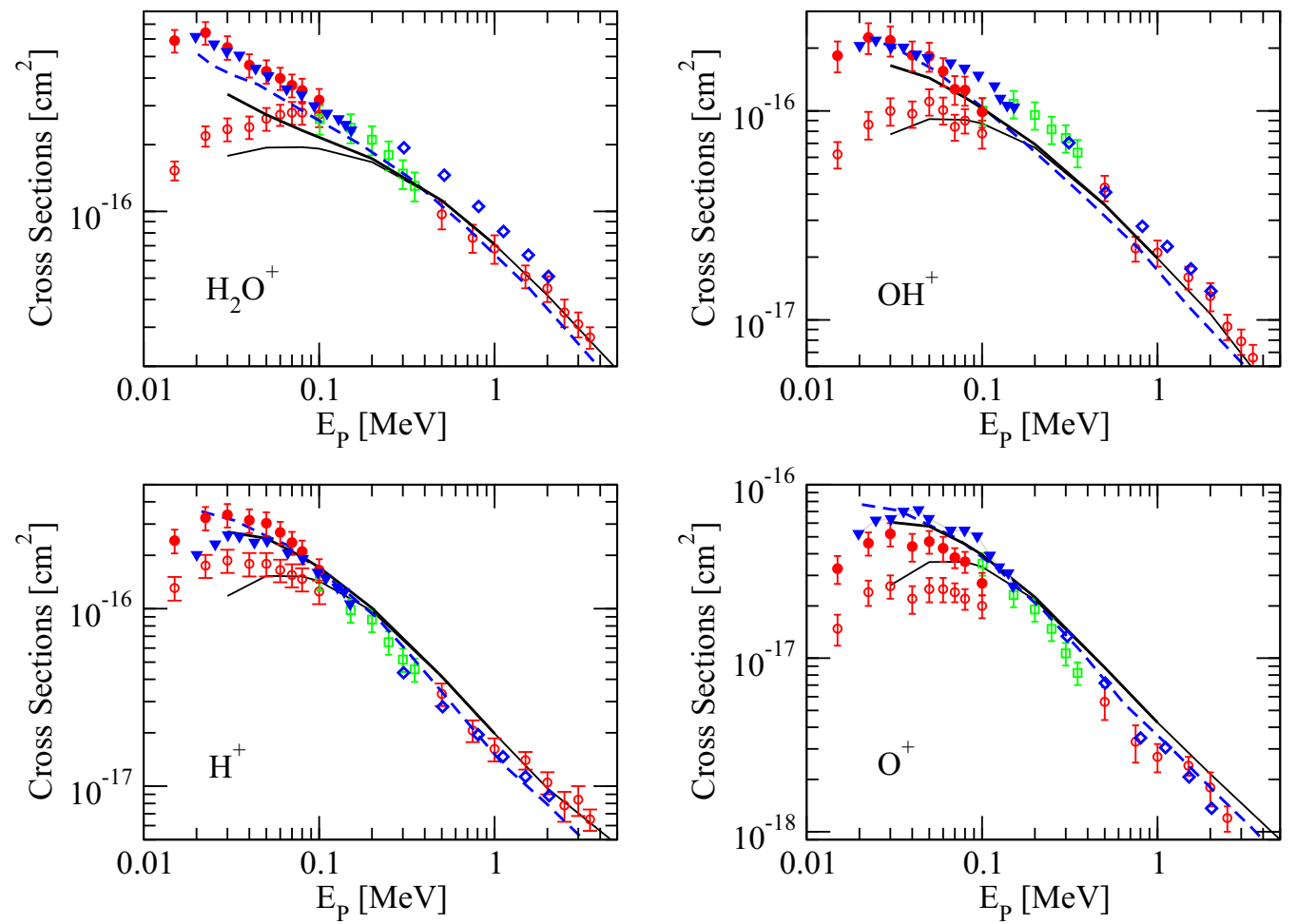

FIG. 6. Fragmentation cross sections for the $\mathrm{H}_{2} \mathrm{O}$ molecule induced by proton impact. Theory: heavy and thin solid lines are the present CDW-EIS-MO results based on electron-loss and ionization cross sections; dashed lines: BGM results of [27]. Experiments: open symbols are

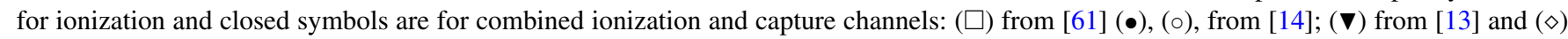
from [16].

Finally let us turn our attention to the double-ionization (DI) process leading to the $\mathrm{OH}^{+}+\mathrm{H}^{+}$and $\mathrm{O}^{+}+\mathrm{H}^{+}$fragmentation channels in the light of the recent measurement of Tavares et al. [16]. The DI can happen by the sequential removal of two electrons (TS2; see [60]) or by the single ionization of $\mathrm{H}_{2} \mathrm{O}$ followed by an Auger-like deexcitation mechanism. Tavares et al. [16] compared their measured DI cross sections with the calculation of Murakami et al. [27], accounting for only the TS2 mechanism, and the observed discrepancy at high impact energies have made them conclude that the Augerlike process is the dominant mechanism above $750 \mathrm{keV}$. This picture is supported by their semiempirical calculation, where only the Auger-like deexcitation process is taken into account with some adjustable parameters, that reveals a good account with experiment at high impact energies. This is well seen in Fig. 7 where these results are presented together with our CDW-EIS-MO calculations. Following Murakami et al. [27], we calculate the cross sections for the $\mathrm{OH}^{+}+\mathrm{H}^{+}$and $\mathrm{O}^{+}+$ $\mathrm{H}^{+}$fragmentation channels as

$$
\begin{gathered}
\sigma_{\mathrm{H}^{+} \mathrm{OH}^{+}}=0.60 \sigma_{D}, \\
\sigma_{\mathrm{H}^{+} \mathrm{O}^{+}}=0.20 \sigma_{D},
\end{gathered}
$$
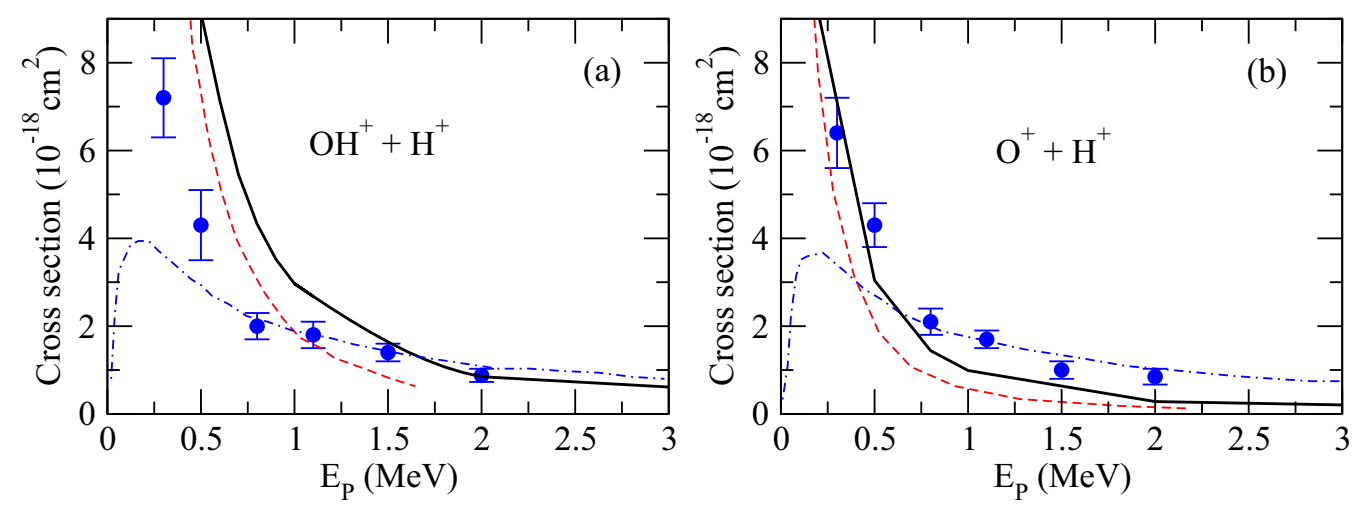

FIG. 7. Cross sections for the fragmentation channels of the doubly ionized $\mathrm{H}_{2} \mathrm{O}$ molecule induced by proton impact. Theory: heavy solid lines are the present CDW-EIS-MO results for the TS2 transition; dashed lines: BGM results of [27]; dot-dashed lines: semiempirical calculation from [16]. Experiments: (•), from [16]. 
where $\sigma_{D}$ is the DI cross section in CDW-EIS-MO and so only the TS2 mechanism is considered. It is interesting to see that the CDW-EIS-MO gives a good description of the experiment for the $\mathrm{OH}^{+}+\mathrm{H}^{+}$dissociation channel at high impact energies. That is for this fragmentation we can interpret the experiment without considering the Auger transition mechanism. For the case of $\mathrm{O}^{+}+\mathrm{H}^{+}$dissociation channels, the cross section in the CDW-EIS-MO is about half of the experimental data for $1 \leqslant E_{P} \leqslant 2 \mathrm{MeV}$. Hence, for the $\mathrm{OH}^{+}+\mathrm{H}^{+}$and $\mathrm{O}^{+}+\mathrm{H}^{+}$fragmentation channels our results do not indicate that the double ionization is dominated by Auger decay, in contrast to the conclusion of Tavares et al. [16]. Also, the interference between the two mechanisms cannot be ruled out. Such a dominant role for the Auger-like process might happen at much higher collision energies. Of course interference between the two mechanisms cannot be ruled out. Unfortunately, the upper limit of the energy range in the experiment seems too low to justify the statement. Also extension of the experiment to lower impact energies would be welcomed to judge the real contribution of the TS2 mechanism.

\section{SUMMARY AND CONCLUSIONS}

We have applied the CDW-EIS model extended for molecular collisions [30] to describe interaction of water with energetic proton projectiles. The evaluated total cross sections for ionization show a good account with the available experimental and theoretical data at high impact energies. Discrepancies between theoretical models appear at medium impact energies, where the cross sections have maxima, however, similar deviations and large error bars of the experimental values do not help to clarify the picture. Electron-capture cross sections are also derived by using the continuation of ionization quantities across the ionization threshold. The evaluated cross-section data show a nice account of the experimental results even at the lowest impact energies, where the CDW-EIS method has limited accuracy.

Having the tools for evaluating electron transition probabilities, multiple ionization, and electron-loss cross sections are calculated within the framework of independent electron picture. Using dissociation schemes and cross sections for $q$-fold electron transitions, cross sections for various fragment ions are determined. It can be concluded that the mechanism of fragmentation is well described by the theories at high impact energies. At the lower impact energies the observed discrepancies might indicate inaccuracies in evaluating single electron transition quantities in perturbative treatments or the breakdown of the independent particle picture.

\section{ACKNOWLEDGMENTS}

This work was supported by the Hungarian Scientific Research Found (Grant No. K109440). We wish to thank Dr. I. Tóth (Babes-Bolyai University) for discussion on the evaluation of molecular wave functions.
[1] Advances in Quantum Chemistry, edited by D. Belkić (Elsevier, Amsterdam, 2013), Vol. 65, pp. 1-449.

[2] B. C. Garett et al., Chem. Rev. 105, 355 (2005).

[3] A. Brahme, Int. J. Radiat. Oncol., Biol., Phys. 58, 603 (2004).

[4] U. Amaldi and G. Kraft, Rep. Prog. Phys. 68, 1861 (2005).

[5] H. Chang, W. Oehrl, P. Elsner, and J. J. Thiele, Free Radical Res. 37, 655 (2003).

[6] B. Boudaiffa, P. Cloutier, D. Hunting, M. A. Hues, and L. Sanche, Science 287, 1658 (2000).

[7] C. Champion, M. Galassi, P. Weck, O. Fojón, J. Hanssen, and R. Rivarola, in Radiation Damage in Biomolecular Systems, edited by G. García Gómez-Tejedor and M. C. Fuss (Springer, New York, 2012), pp. 263-289.

[8] L. H. Tuboren and W. E. Wilson, J. Chem. Phys. 66, 5202 (1997).

[9] C. D. Cappello, C. Champion, O. Boudrioua, H. Lekadir, Y. Sato, and D. Ohsawa, Nucl. Instrum. Methods Phys. Res., Sect. B 267, 781 (2009).

[10] S. Nandi, S. Biswas, A. Khan, J. M. Monti, C. A. Tachino, R. D. Rivarola, D. Misra, and L. C. Tribedi, Phys. Rev. A 87, 052710 (2013).

[11] M. E. Rudd, T. V. Goffe, R. D. DuBois, and L. H. Toburen, Phys. Rev. A 31, 492 (1985).

[12] M. A. Bolorizadeh and M. E. Rudd, Phys. Rev. A 33, 888 (1986).

[13] F. Gobet, S. Eden, B. Coupier, J. Tabet, B. Farizon, M. Farizon, M. J. Gaillard, M. Carré, S. Ouaskit, T. D. Märk et al., Phys. Rev. A 70, 062716 (2004).

[14] H. Luna, A. L. F. de Barros, J. A. Wyer, S. W. J. Scully, J. Lecointre, P. M. Y. Garcia, G. M. Sigaud, A. C. F. Santos, V. Senthil, M. B. Shah et al., Phys. Rev. A 75, 042711 (2007).
[15] K. H. Tan and C. E. Brion, Chem. Phys. 29, 299 (1978).

[16] A. C. Tavares, H. Luna, W. Wolff, and E. C. Montenegro, Phys. Rev. A 92, 032714 (2015).

[17] M. Bernal and J. Liendo, Nucl. Instrum. Methods Phys. Res., Sect. B 262, 1 (2007).

[18] O. Boudrioua, C. Champion, C. Dal Cappello, and Y. V. Popov, Phys. Rev. A 75, 022720 (2007).

[19] C. Champion and C. D. Cappello, Nucl. Instrum. Methods Phys. Res., Sect. B 267, 881 (2009).

[20] S. Houamer, Y. Popov, C. D. Cappello, and C. Champion, Nucl. Instrum. Methods Phys. Res., Sect. B 267, 802 (2009).

[21] G. H. Olivera, P. D. Fainstein, and R. D. Rivarola, Phys. Med. Biol. 41, 1633 (1996).

[22] G. H. Olivera, C. Caraby, P. Jardin, A. Cassimi, L. Adoui, and B. Gervias, Phys. Med. Biol. 43, 2347 (1998).

[23] C. A. Tachino, J. M. Monti, O. A. Fojón, C. Champion, and R. D. Rivarola, J. Phys. B: At., Mol. Opt. Phys. 47, 035203 (2014).

[24] B. Gervais, M. Beuve, G. Olivera, and M. Galassi, Radiat. Phys. Chem. 75, 493 (2006).

[25] H. Lekadir, I. Abbas, C. Champion, and J. Hanssen, Nucl. Instrum. Methods Phys. Res., Sect. B 267, 1011 (2009).

[26] C. Illescas, L. F. Errea, L. Méndez, B. Pons, I. Rabadán, and A. Riera, Phys. Rev. A 83, 052704 (2011).

[27] M. Murakami, T. Kirchner, M. Horbatsch, and H. J. Lüdde, Phys. Rev. A 85, 052704 (2012).

[28] M. Murakami, T. Kirchner, M. Horbatsch, and H. J. Lüdde, Phys. Rev. A 86, 022719 (2012).

[29] M. J. Frisch et al., Gaussian 09 Revision A.1 (Gaussian Inc., Wallingford, CT, 2009). 
[30] L. Gulyás, I. Tóth, and L. Nagy, J. Phys. B: At., Mol. Opt. Phys. 46, 075201 (2013).

[31] R. Hoffmann, J. Chem. Phys. 39, 1397 (1963).

[32] G. Gerzberg, Molecular Spectra and Molecular Structure, III. Electronic Spectra and Electronico Polyatomic Molecule (Van Nostrad, Princeton, NJ, 1966).

[33] W. J. Hehre, R. F. Stewart, and J. A. Pople, J. Chem. Phys. 51, 2657 (1969).

[34] I. Tóth, R. Campeanu, V. Chis, and L. Nagy, Phys. Lett. A 360, 131 (2006).

[35] L. Gulyás, P. D. Fainstein, and A. Salin, J. Phys. B 28, 245 (1995).

[36] D. S. F. Crothers and L. J. Dube, in Advances in Atomic, Molecular, and Optical Physics, edited by D. Bates and B. Bederson (Academic Press, Boston, San Diego, 1992), Vol. 30, p. 287.

[37] N. Stolterfoht, R. D. DuBois, and R. D. Rivarola, Electron Emission in Heavy Ion-Atom Collisions (Springer, Berlin, 1997).

[38] D. S. F. Crothers, J. Phys. B: At., Mol. Opt. Phys. 15, 2061 (1982).

[39] D. S. F. Crothers and J. F. McCann, J. Phys. B: At., Mol. Opt. Phys. 16, 3229 (1983).

[40] D. Belkić, R. Gayet, and A. Salin, Phys. Rep. 56, 279 (1979).

[41] P. D. Fainstein, L. Gulyás, and A. Salin, J. Phys. B: At., Mol. Opt. Phys. 29, 1225 (1996).

[42] L. Gulyás, A. Igarashi, P. D. Fainstein, and T. Kirchner, J. Phys. B: At., Mol. Opt. Phys. 41, 025202 (2008).

[43] A. R. Edmonds, Angular Momentum in Quantum Mechanics (Princeton University Press, Princeton, NJ, 1957).

[44] M. Horbatsch, Phys. Lett. 187, 185 (1994).

[45] M. Rodbrø and F. D. Andersen, J. Phys. B: At., Mol. Opt. Phys. 12, 2883 (1979).
[46] J. Burgdörfer, in Lecture Notes in Physics, edited by K. O. Groeneweld, W. Meckbach, and I. A. Sellin (Springer, Berlin, 1984), Vol. 213, p. 32.

[47] L. J. Dube and A. Salin, J. Phys. B: At., Mol. Opt. Phys. 20, L499 (1987).

[48] L. Gulyás, A. Igarashi, and T. Kirchner, J. Phys. B: At., Mol. Opt. Phys. 45, 085205 (2012).

[49] V. Chobut, Y. Frongillo, J. Patau, T. Goulet, M.-J. Fraser, and J. Jay-Gerin, Radiat. Phys. Chem. 51, 229 (1998).

[50] D. P. Dewangan and J. Eichler, Phys. Rep. 247, 59 (1994).

[51] K. Siegbahn, ESCA Applied to Free Molecules (North-Holland, Amsterdam, 1969).

[52] B. Senger and R. V. Rechemann, Nucl. Instrum. Methods Phys. Res., Sect. B 2, 204 (1984).

[53] M. E. Galassi, R. D. Rivarola, M. Beuve, G. H. Olivera, and P. D. Fainstein, Phys. Rev. A 62, 022701 (2000).

[54] H. J. Lüdde, T. Spranger, M. Horbatsch, and T. Kirchner, Phys. Rev. A 80, 060702 (2009).

[55] L. H. Toburen, M. Y. Nakai, and R. A. Langley, Phys. Rev. 171, 114 (1968).

[56] L. Gulyás, A. Igarashi, and T. Kirchner, Phys. Rev. A 86, 024701 (2012).

[57] L. Gulyás, P. D. Fainstein, and T. Shirai, Phys. Rev. A 65, 052720 (2002),

[58] P. Fainstein, G. Olivera, and R. Rivarola, Nucl. Instrum. Methods Phys. Res., Sect. B 107, 19 (1996).

[59] T. Kirchner, L. Gulyás, R. Moshammer, M. Schulz, and J. Ullrich, Phys. Rev. A 65, 042727 (2002).

[60] J. H. McGuire, Electron Correlation Dynamics in Atomic Collisions (Cambridge University Press, Cambridge, England, 1997).

[61] U. Werner, K. Beckord, J. Becker, and H. O. Lutz, Phys. Rev. Lett. 74, 1962 (1995). 